\title{
Temporal predictability does not impact attentional blink performance: Effects of fixed vs. random inter-trial intervals
}

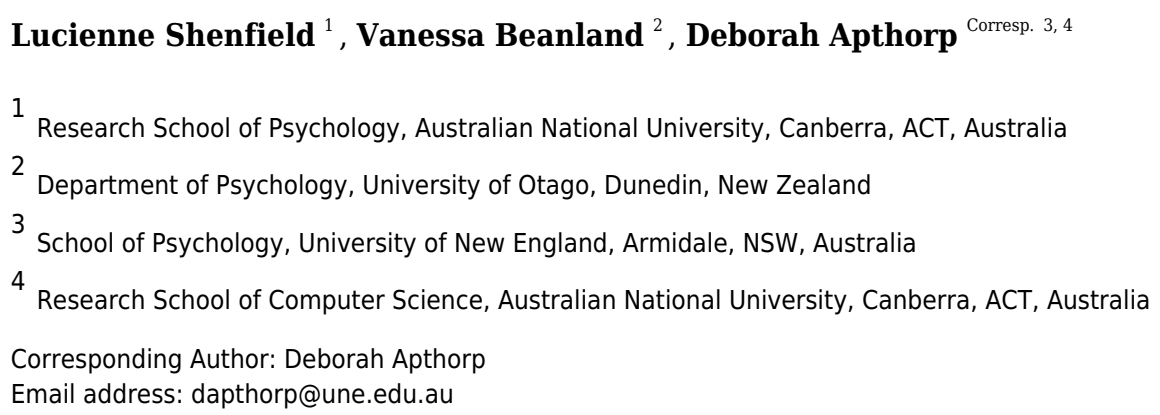

Background: Does the inclusion of a randomized inter-trial interval (ITI) impact performance on an Attentional Blink ( $A B)$ task? The $A B$ phenomenon is often used as a test of transient attention (Dux \& Marois, 2009); however, it is unclear whether incorporating aspects of sustained attention, by implementing a randomized ITI, would impact task performance. The current research sought to investigate this, by contrasting a standard version of the $A B$ task with a random ITI version to determine whether a performance change, reflecting a change in difficulty, engagement, or motivation. Method: 30 university students ( 21 female; age range $18-57, M_{\text {age }}=21.5, S D=7.4$ ) completed both versions of the task, in counterbalanced order. Results: No significant difference in performance was found between the standard $A B$ task and the $A B$ task with the random ITI. Bayesian analyses suggested moderate evidence for the null. Conclusion: Temporal unpredictability did not appear to impact task performance. This suggests that the standard $A B$ task has cognitive properties with regards to task difficulty, engagement, and motivation, that are inherently similar to tasks that employ a randomized ITI to measure sustained attention (i.e. the Psychomotor Vigilance Task; PVT; Dinges \& Powell, 1985). This finding provides important support for future research which may seek to obtain a more detailed understanding of attention through the comparison of performance on transient and sustained attention tasks. 
1. Temporal predictability does not impact 2 attentional blink performance:

3 Effects of fixed vs. random inter-trial

${ }_{4}$ intervals

5

6 Lucienne Shenfield ${ }^{1}$, Vanessa Beanland ${ }^{2}$, Deborah Apthorp ${ }^{3,4}$,

$8 \quad{ }^{1}$ Research School of Psychology, Australian National University, Canberra, ACT 2601,

9 Australia

$10 \quad{ }^{2}$ Department of Psychology, University of Otago, Dunedin, New Zealand

$11{ }^{3}$ School of Psychology, University of New England, Armidale, NSW, Australia

$12{ }^{4}$ Research School of Computer Science, Australian National University, Canberra, ACT,

13 Australia

14

15 Corresponding Author:

16 Deborah Apthorp ${ }^{3,4}$

17 School of Psychology, University of New England, NSW 2351, Australia

18 Email address: dapthorp@une.edu.au

19 


\section{Abstract}

23 Background: Does the inclusion of a randomised inter-trial interval (ITI) impact performance

24 on an Attentional Blink (AB) task? The $\mathrm{AB}$ phenomenon is often used as a test of transient

25 attention (Dux \& Marois, 2009); however, it is unclear whether incorporating aspects of

26 sustained attention, by implementing a randomised ITI, would impact task performance. The

27 current research sought to investigate this, by contrasting a standard version of the AB task with

28 a random ITI version to determine whether a performance changed, reflecting a change in

29 difficulty, engagement, or motivation. Method: 30 university students (21 female; age range 18-

$\left.3057, M_{\text {age }}=21.5, S D=7.4\right)$ completed both versions of the task, in counterbalanced order.

31 Results: No significant difference in performance was found between the standard AB task and

32 the AB task with the random ITI. Bayesian analyses suggested moderate evidence for the null.

33 Conclusion: Temporal unpredictability did not appear to impact task performance. This

34 suggests that the standard $\mathrm{AB}$ task has cognitive properties with regards to task difficulty,

35 engagement, and motivation, that are inherently similar to tasks which employ a randomised ITI

36 to measure sustained attention (i.e. the Psychomotor Vigilance Task; PVT; Dinges \& Powell,

37 1985). This finding provides important support for future research which may seek to obtain a

38 more detailed understanding of attention through the comparison of performance on transient and

39 sustained attention tasks. 


\section{Introduction}

42 Given the important role attention plays in behavioural outcomes (Carrasco, 2011; Correa,

43 Lupiáñez, Madrid, \& Tudela, 2006), many researchers have sought to better understand

44 attentional processes by examining performance limitations within specific attentional tasks

45 (Raz, 2004). This research has encompassed a variety of paradigms and tasks, exploring both

46 spatial (e.g., Intriligator \& Cavanagh, 2001) and temporal (e.g., Potter, Wyble, Hagmann, \&

47 McCourt, 2013) characteristics of attention. The present research focuses on the attentional blink

48 (AB), which has been used to investigate temporal aspects of attentional selection (Dux \&

49 Marois, 2009).

The $\mathrm{AB}$ occurs when an observer has a reduced ability to perceive a second target (T2)

51 within a set of distractors when it is presented within $800 \mathrm{~ms}$ of an initial target (T1; see Figure

52 1). The phenomenon was first documented by Broadbent and Broadbent (1987); however, the term 'attentional blink' was coined by Raymond, Shapiro, and Arnell (1992). This name does not

54 refer to a physical blink, but rather to the momentary lapse in attentional ability after selection of

55 T1 (Shapiro, Raymond, \& Arnell, 1997). The event is typically measured using a Rapid Serial

56 Visual Presentation (RSVP) task (Potter \& Levy, 1969). During the task, stimuli are displayed in

57 quick succession, usually around $100 \mathrm{~ms}$ apart, in order to test information processing limits.

58 Importantly, Raymond et al. noted that detection of T2 significantly improved when subjects

59 were instructed to deliberately ignore $\mathrm{T} 1$. This suggests that the $\mathrm{AB}$ occurs due to attentional, as

60 opposed to sensory, limitations, validating its use as a measure of attentional performance

61 (Raymond et al., 1992). The attentional nature of the effect has been further confirmed through

62 numerous of AB studies over the past 25 years (see Dux \& Marois, 2009, for a review).

63 [INSERT FIGURE 1 ABOUT HERE] 
Past research has investigated many factors thought to influence performance on the $\mathrm{AB}$

66

67 68

task, including individual differences (Colzato, Spapé, Pannebakker, \& Hommel, 2007; Kelly \& Dux, 2011; MacLean, Arnell, \& Cote, 2012; Martens, Munneke, Smid, \& Johnson, 2006). Although individual differences are strongly predictive of $\mathrm{AB}$ magnitude, recent research suggests that levels of anticipation can also affect AB performance (Maclean \& Arnell, 2011). This research has shown that changing temporal characteristics, particularly relating to temporal predictability, can affect blink magnitude. For instance, Maclean and Arnell (2011) found that increased attentional blink magnitude was associated with higher levels of anticipatory attention, as measured by alpha oscillations using electroencephalogram (EEG). This is consistent with the theory that the $\mathrm{AB}$ reflects overinvestment in $\mathrm{T} 1$ (i.e., excessive anticipation), which leaves the observer unprepared to process T2. Increasing temporal predictability of the AB sequence may therefore attenuate the $\mathrm{AB}$ by allowing observers to better prepare for when to allocate attentional resources. There are several aspects of stimulus timing that can be manipulated in an AB task, including foreperiod, target onset asynchrony (TOA) and inter-trial interval (ITI).

\section{Trial Foreperiod}

In the context of $\mathrm{AB}$, foreperiod refers to the time between trial commencement and presentation of T1. The impact of foreperiod on AB was investigated by Badcock, Badcock, Fletcher, and Hogben (2013), who manipulated the foreperiod by extending, shortening and/or randomising the time between trial onset and T1 presentation. Badcock et al. (2013) found that, compared with randomly variable foreperiods, having a predictable foreperiod attenuated the $\mathrm{AB}$, but only for relatively long foreperiods ( $\sim 880 \mathrm{~ms})$. They concluded that brief or temporally unpredictable foreperiods contribute to the $\mathrm{AB}$, as observers do not have adequate time to 
87 prepare for the dual task of detecting both T1 and T2 (Badcock et al., 2013). The result is that

88 observers prioritise processing of $\mathrm{T} 1$, and require additional time to fully process $\mathrm{T} 2$, which

89 results in an $\mathrm{AB}$ if $\mathrm{T} 2$ follows shortly after $\mathrm{T} 1$.

\section{Target Onset Asynchrony (TOA)}

91 TOA (also called stimulus onset asynchrony or SOA) refers to the length of time in milliseconds

92 between the presentation of $\mathrm{T} 1$ and $\mathrm{T} 2$. In AB tasks this is commonly referred to as the "lag",

93 which denotes the number of distractors between $\mathrm{T} 1$ and T2. Lag is arguably the strongest

94 predictor of $\mathrm{T} 2$ perception: $\mathrm{T} 2$ is reliably perceived when presented immediately after $\mathrm{T} 1$ (i.e.,

95 lag 1), making it resistant to the $\mathrm{AB}$ effect. This is known as lag 1 sparing, a robust feature of the

96 AB (Hommel \& Akyürek, 2005; Lunau \& Olivers, 2010; Martin \& Shapiro, 2008; although see

97 Visser et al., 1999). The ability to perceive T2 is typically worst at around lag 3, and steadily

98 improves until around lag 8 , when it is usually reliably perceived again (see Figure 2).

[INSERT FIGURE 2 ABOUT HERE]

101

102 Introducing a consistent lag or TOA (e.g., presenting a block of only lag 3 trials) improves $\mathrm{AB}$

103 task performance, especially when participants are made explicitly aware of the consistency

104 (Martens \& Johnson, 2005; Visser, 2015; Visser, Tang, Badcock, \& Enns, 2014). Even when lag

105 varies within blocks, providing a lag length cue before the commencement of each trial can

106 reduce blink magnitude (Martens \& Johnson, 2005). This further demonstrates the important role

107 that anticipation plays in $\mathrm{AB}$ performance, with some researchers suggesting that familiarity with

108 these patterns may account for training improvements (Tang, Badcock, \& Visser, 2014), and

109 even learning effects (Choi, Chang, Shibata, Sasaki, \& Watanabe, 2012). 


\section{Inter-Trial Interval (ITI)}

111 ITI refers to the time between a participant's response on a trial N-1, and the start of the

112 next trial N. Although AB tasks classically employ a fixed ITI, the present research seeks to

113 explore the role that the ITI plays in shaping task performance. The time between one trial

114 ending and the next trial commencing may be crucial with regard to preparation and anticipation,

115 and as yet these intervals have not been investigated in relation to the AB. This would be

116 particularly relevant to research investigating the role of pre-stimulus alpha (Maclean \& Arnell,

117 2011; Maclean, Arnell, \& Cote, 2012; Zauner et al., 2012).

118 Although historically it has been assumed that ITI does not affect AB performance, ITI

119 has been found to influence both reaction time and accuracy in vigilance tasks, such as the

120 Psychomotor Vigilance Task (PVT; Dinges \& Powell, 1985) and the Continuous Performance

121 Task (CPT; Cornblatt, Risch, Faris, Friedman, \& Erlenmeyer-Kimling, 1988). This raises the

122 question of whether ITI can also affect performance on the $\mathrm{AB}$, a temporal attention task which

123 usually requires unspeeded responses.

\section{The Current Study}

125 Previous studies investigating the temporal predictability of the AB have manipulated

126 temporal attributes within the RSVP stream. Usually this is achieved by identifying a temporal

127 aspect which is typically variable (e.g., TOA or foreperiod), and applying a fixed continuous

128 duration, to see if this attenuates the AB. However, the current research seeks to investigate this

129 by taking a temporal attribute of the task which is usually fixed (ITI) and varying it, to see if

130 greater variability increases the $\mathrm{AB}$ by reducing temporal predictability, thus lessening readiness

131 for the task. 


\section{Method}

\section{Participants}

134 Thirty observers (21 female; age range $\left.18-57, M_{\mathrm{age}}=21.5, S D=7.4\right)$ provided written

135 informed consent and participated voluntarily. Most were psychology undergraduates who were

136 received course credit. Ethical aspects of the research were approved by the Australian National

137 University Human Research Ethics Committee (protocol 2015/184), in accordance with the

138 ethical standards of the 2008 Declaration of Helsinki.

139 Design

140 A repeated-measures design was used. Participants completed both versions of the AB

141 task in counterbalanced order in a single 30-minute session.

\section{Apparatus}

143 Stimuli were presented on a 23.6 inch VIEWPixx liquid crystal display (LCD) with a

144 refresh rate of $120 \mathrm{~Hz}$ and a resolution of 1920 x 1080 pixels. Behavioural responses for the AB

145 were collected using a Cedrus RB-8 SUBJECT response box, with buttons labelled 2, 3, 4, 7, 8,

146 and 9. Two buttons were left blank to enable participants to indicate if T2 did not appear or they

147 did not perceive it. Experimental stimuli were created using Psychophysics Toolbox (version 3)

148 for MATLAB R2012b (Brainard, 1997; Pelli, 1997; Kleiner et al., 2007).

149 Stimuli

150 Stimuli were displayed in white (luminance $\left.96.2 \mathrm{~cd} / \mathrm{m}^{2}\right)$ on a grey $\left(17.3 \mathrm{~cd} / \mathrm{m}^{2}\right.$ )

151 background. Items were presented using an RSVP stream in $1.23^{\circ}$ Helvetica font. Each trial

152 commenced with a fixation cross and contained between 18 and 22 stimuli. Targets were

153 numerals $(2,3,4,7,8,9)$ and distractors were uppercase letters, excluding those that could be

154 confused with numbers (i.e., I, L, O, Q). Each stimulus in the stream appeared for exactly $100 \mathrm{~ms}$ 
155 (12 frames at $120 \mathrm{~Hz}$ ). Most trials included two targets (T1 and T2), with some 'blank' trials

156 including only one target. T1 appeared with a jitter of \pm 2 items, after 4-8 distractors, and T2 was

157 presented at either lag 3 or lag 8 . In both conditions, 30 trials were presented for each lag, with

158 lags randomly interleaved. When each $\mathrm{AB}$ trial had concluded, participants were allocated 3.5

159 seconds in which to respond. If participants did not respond within the time frame, a non-

160 response was recorded, and the next trial commenced. The ITI commenced immediately after the

161 participant had keyed in their answer for T2, or after the time limit had elapsed.

162 During the fixed-ITI condition, the next trial always commenced 1500 milliseconds after the

163 participant's previous response had been made. During the random-ITI condition, ITI varied

164 randomly between 100 and 3100 milliseconds after the previous response had been made. The

165 random interval was set using MATLAB's internal rand.m function and PsychToolbox's

166 "WaitSecs.m" (see code section of the Supplementary Materials).

\section{Procedure}

168 Participants were given two AB practice blocks with auditory feedback. Both practice

169 trials used a fixed ITI. A high-pitched beep indicated that the participant gave the correct

170 response, while a low-pitched beep indicated that the participant gave an incorrect response. The

171 first practice block involved 12 trials at half speed (24 frames per second). The second practice

172 block was at full speed. Following this, participants completed the two experimental blocks in

173 counterbalanced order. Experimental trials took place in a darkened room to avoid peripheral

174 distractions. To ensure consistency and minimise distraction, the experimenter was not present

175 during the experimental trials. After completing both versions of the task, participants were

176 asked verbally if they had detected any difference between the two versions of the task. 
177 Qualitative verbal responses were recorded. After answering this question, participants were

178 verbally debriefed.

179 Statistical Analysis

180 Three dependent variables were analysed: T1 accuracy, T2|T1 accuracy, and blink

181 magnitude. Of these, blink magnitude was the primary dependent variable of interest. T2|T1

182 accuracy represents accuracy at identifying T2 on trials where T1 was correctly identified, and

183 was used to calculate blink magnitude. T2|T1 was compared between lags to confirm that an AB

184 effect was observed. Blink magnitude was calculated by subtracting T2|T1 at lag 3 from T2|T1 at

185 lag $8\left(\mathrm{~T} 2\left|\mathrm{~T} 1_{\operatorname{lag} 8}-\mathrm{T} 2\right| \mathrm{T} 1_{\operatorname{lag} 3}\right)$, and was compared between fixed-ITI and random-ITI conditions.

186 T1 accuracy was also compared between conditions, to ensure that the blink magnitude results

187 could not be attributable to systematic differences in T1 identification. Statistical analyses were

188 conducted in jamovi, Version 9.5 .15 (jamovi project, 2018), and non-parametric tests were used

189 where Shapiro-Wilk tests indicated assumptions of normality were violated. Verbal responses to

190 the detection question were examined to determine whether the experimental manipulation had

191 successfully evaded detection.

192 In addition, we included supplemental Bayesian analyses of the results, in order to give

193 more information about null results. Unlike traditional NHST, Bayesian analyses can give

194 information about the extent to which the data support the null hypothesis (Quintana \& Williams,

195 2018). When evaluating support for the null in a Bayesian framework, it is more intuitive to use

$196 \mathrm{BF}_{01}$ (the simple inverse of $\mathrm{BF}_{10}$ ), which in simple terms gives an odds ratio of how much more

197 likely the null is than the alternative hypothesis (e.g. a value of $\mathrm{BF}_{01}=3.5$ would indicate that the

198 null is 3.5 times more likely than the alternative; Quintana \& Williams, 2018). These analyses

199 were carried out in JASP statistical software (JASP team, 2019), which provides a free, easy-to- 
200 use interface for these analyses. All data and analyses are available as supplementary materials to 201 the paper.

\section{Results}

203 T1 Accuracy

Average T1 accuracy, expressed as proportion correct, was $.88(S D=.10$, median $=.89)$

205 for the fixed-ITI condition and $.87(S D=.11$, median $=.88)$ for the random-ITI condition. A

206 Wilcoxon Signed Rank Test indicated there was no systematic difference in T1 accuracy

207 between conditions, $W=167, p=.829, M($ diff $)=.01, S E($ diff $)=.03,95 \% \mathrm{CI}=[-.04, .03], \delta=$

208 .07. In addition, we carried out a Bayesian paired-samples t-test analysis using JASP (JASP

209 Team, 2019), using a Cauchy prior of .707. This provided moderate evidence for the null, $B F_{01}=$

2104.79 , error $\%=.009$.

\section{Attentional Blink}

Figure 3 displays mean T2|T1 accuracy for lags 3 and 8 in each condition. Wilcoxon

213 Signed Rank Tests confirmed there was a significant AB effect (i.e., identification of T2 was

214 consistently lower for lag 3 compared with lag 8) in both the fixed-ITI condition, $W=465, p<$

215.001 , mean difference $=.17, S E($ diff $)=.03,95 \% C I=[.11, .23], \delta=1.02$, and the random-ITI

216 condition, $W=446, p<.001, M($ diff $)=.15, S E($ diff $)=.03,95 \% C I=[.10, .21], \delta=1.02$.

[INSERT FIGURE 3 ABOUT HERE]

221 indicated that blink magnitude did not significantly differ between fixed-ITI $(M=.18 S D=$ $.14 \%)$ and random-ITI $(M=.16, S D=.16)$ blocks, $t(29)=.49, p=.631, M($ diff $)=.01, S E($ diff $)=$ 
$223.03,95 \% C I=[-.04, .07], \delta=.09$. A follow-up Bayesian paired-samples t-test using JASP

224 showed moderate evidence for the null, using a Cauchy prior of .707, $B F_{01}=4.61$, error $\%=$ $225 \quad .007$.

\section{Order effects}

227

228

229

230

231

232

233

234

235

236

237

238

239

240

241

242

243

244

245

As the trial order was randomised (either random ITI first or fixed ITI first), order effects were analysed in the results using a mixed ANOVA on blink magnitude, with ITI as the withinsubjects factor and order as the between-subjects factor. There was no significant main effect of ITI (fixed vs. random), $F(1,21)=.3, p=\mathrm{p}=.617, \eta_{p}^{2}=.009$, or order, $F(1,28)=1.7, p=.202, \eta_{p}^{2}$ $=.057$, and no significant interaction between trial order and ITI, $F(1,28)=3.5, p=.072, \eta_{p}^{2}=$

.11. However, it should be noted that this analysis had low power, as it was not initially included as part of our experimental design, so would be worth exploring in further research. The results are plotted in Figure 4.

\section{[INSERT FIGURE 4 ABOUT HERE]}

\section{Reaction times}

Since reaction times are occasionally used as an indirect measure of task engagement (e.g. see Brigadoi et al., 2018), we also analysed the participants' reaction times for the first button press response in the task. Even though responses were not speeded, slower reaction times in the random-ITI condition might reflect increased task difficulty or reduced attention to the task. Reaction times were computed as median RT from the end of the RSVP stream to the first button press (response for T1) across both lags. A paired t-test (used since assumptions of normality were not violated) indicated that median reaction time (in seconds) did not significantly differ between fixed-ITI $(M=1.001 S D=.199)$ and random-ITI $(M=1.009, S D=$ $.189)$ blocks, $t(29)=.30, p=.769, M($ diff $)=.008, S E($ diff $)=.026,95 \% C I=[-.045, .06], \delta=$ 
246 .05. A follow-up Bayesian paired-samples t-test using JASP showed moderate evidence for the

247 null, using a Cauchy prior of $.707, B F_{01}=4.94$, error $\%=.011$.

\section{Subjective Reports}

249 Qualitative verbal responses showed that 9 out of 30 participants did not detect any

250 difference between fixed- and random-ITI blocks. An additional 19 participants identified

251 incorrect differences (e.g., change in overall length, change in task stimuli). Only two

252 participants correctly detected that one version of the task had randomised intervals between

253 trials; one of these participants had extensive prior exposure of the original AB task through

254 other research participation. This suggests that most participants were not consciously aware of

255 the temporal difference between AB blocks.

\section{Discussion}

This finding does not show evidence that randomising the ITI affects performance in the

258

259

260

261

262

263

264

265

266
AB task. A post-hoc Bayesian analysis shows moderate evidence for the null. This result expands upon findings made by Badcock et al. (2013) who found that randomising the ISI between trial commencement and $\mathrm{T} 1$ presentation did not affect $\mathrm{AB}$ performance when compared to an extended ISI $(\sim 880 \mathrm{~ms})$. The comparison of a random ITI with a fixed ITI (such as is more typically employed in a standard $\mathrm{AB}$ task), provides useful information regarding the attentional resources required to perform both tasks. The maintenance of accuracy during the random ITI condition suggests that these resources do not vary between conditions. In addition, the lack of differences in the reaction time data suggest that participants were most likely equally engaged during both types of task. Qualitatively, stable performance across the two trial types

Peer] reviewing PDF | (2019:10:42318:1:1:NEW 29 Jan 2020) 
267 suggests that overall task difficulty, engagement, and motivation do not deviate when a

268 randomised ITI is present.

269 Our analysis on order effects in the data was suggested by a reviewer, but since it was a

270 post-hoc analysis we note that it is somewhat underpowered, so these results should be

271 interpreted with caution. However, it is interesting to note that the learning effect from the first to

272 the second session seems somewhat less in the random-first compared to the fixed-first group

273 (see Figure 4). This would be an interesting trend to follow up in future studies.

274 These findings have useful implications for future research, as it provides confirmation

275 that the standard AB task, typically used as a measure of transient attention, has similar levels of

276 interest and difficulty to those tasks which employ a random ITI. Such tasks are typically used

277 as a measure of sustained attention (e.g. the Psychomotor Vigilance Test (PVT); Dinges \&

278 Powell, 1985 and the Continuous Performance Test (CPT); Cornblatt, Risch, Faris, Friedman, \&

279 Erlenmeyer-Kimling, 1988). The current finding provides support for future research, which

280 may seek to directly compare sustained and transient attention using the aforementioned tasks,

281 without task difficulty/engagement confounding performance results.

282 The method adopted in the present experiment can be summarised as being a highly

283 pared-back version of the $\mathrm{AB}$ task, in conjunction with a brief but randomised ITI, akin to the

284 PVT, though considerably reduced in length. The decision to use the simplified version of the

$285 \mathrm{AB}$ task was mainly practical; fewer lags required fewer trials overall, increasing the number of

286 trials possible for each lag and thus giving increased signal-to-noise ratio. However, the rationale

287 for the use of the random interval was more complex.

288 Although most experimental paradigms involving a PVT use longer randomised intervals

289 of 2 to 10 seconds, this was deemed too long in the current experiment, which instead used a 
290 randomised ITI of 0.1 to 3.1 seconds. Firstly, the inclusion of a longer ITI would drastically alter

291 the length of the two versions of the task, potentially rendering them incomparable. Secondly, it

292 was believed that the use of a longer ITI might appear too obvious in contrast with the fixed ITI,

293 and that this noticeable difference might consciously influence participants' performance.

294 Indeed, it appeared that the abridged ITI of 0.1 to 3.1 seconds did successfully evade detection in 295 most cases.

296 It is possible that, during the randomised ITI condition, participants may have performed

297 differently during longer compared to shorter ITIs - there is certainly precedent for this in the

298 literature (e.g. see Brigadoi et al., 2018). In addition, performance on each trial might have been

299 affected by the length of the previous trial's ITI (trial n-1); this might be expected from evidence

300 in the foreperiod literature (e.g. see Los, Knol \& Boers, 2001). Unfortunately, it was not possible

301 to carry out these analyses with our current data, as we did not record the exact ITI for each trial.

302 However, we have now updated the experimental code to include this variable, and it is available

303 with the Supplementary Materials to facilitate replication and extension of this study.

$304 \quad$ Further studies could undertake to more clearly establish the perceptual limits associated

305 with the incorporation of a randomised ITI. It is possible that, if imperceptible, a longer

306 randomised ITI might more accurately represent a hybrid version of the PVT and AB tasks, and

307 could therefore more accurately measure the possibility that task difficulty affects performance.

308 Some research suggests much longer randomised ITIs $(6000-10000 \mathrm{~ms})$ had a measurable effect

309 on task performance and engagement in the SNARC effect (the spatial numerical association of

310 response codes; Brigadoi et al., 2018), and indeed the random ITIs in sustained attention tasks do

311 tend to be longer than $3000 \mathrm{~ms}$ (our maximum ITI in the random condition). It would be useful

312 to include these longer ITIs in future studies. 


\section{Conclusion}

314 The current findings may benefit future research which could seek to compare transient

315 attention using the $\mathrm{AB}$ task, with measures of sustained attention that employ a random ITI, such

316 as the PVT or CPT. Randomising the ITI on the AB task did not significantly impact

317 performance, suggesting that both tasks may have similar levels of difficulty. This finding has

318 broad implications for future research, expanding possibilities for comparing the effects of

319 factors, such as level of arousal or affective state on these discrete forms of attention. This is

320 valuable, given the wealth of existing research concerning both the AB and PVT (Dux \& Marois,

321 2009; Langner \& Eickhoff, 2013). Importantly, these findings support the legitimacy of

322 comparing performance on the AB and random ITI tasks as a measure of transient and sustained

323 attention respectively. This finding may be crucial in obtaining a more detailed understanding of

324 attention, as it provides support for future research to investigate the roles of sustained and

325 transient attention in the same individuals, using these tasks.

326

327

328

Peer] reviewing PDF | (2019:10:42318:1:1:NEW 29 Jan 2020) 
329 References

330 Badcock, N. A., Badcock, D. R., Fletcher, J., \& Hogben, J. (2013). The role of preparation time 331 in the attentional blink. Vision research, 76, 68-76. doi: 10.1016/j.visres.2012.10.010

332 Basner, M., \& Dinges, D. F. (2011). Maximizing Sensitivity of the Psychomotor Vigilance Test 333 (PVT) to Sleep Loss. Sleep, 34(5), 581-591.

334 Brainard, D. H. (1997). The psychophysics toolbox. Spatial vision, 10, 433-436.

335 Brigadoi, S., Basso Moro, S., Falchi, R., Cutini, S., \& Dell'Acqua, R. (2018). On pacing trials 336 while scanning brain hemodynamics: The case of the SNARC effect. Psychonomic 337 Bulletin \& Review, 25, 2267-2273.

338 Broadbent, D. E., \& Broadbent, M. H. (1987). From detection to identification: Response to multiple targets in rapid serial visual presentation. Perception \& psychophysics, 42(2), 105-113.

341 Carrasco, M. (2011). Visual attention: the past 25 years. Vision Res, 51(13), 1484-1525. doi: 10.1016/j.visres.2011.04.012

343 Choi, H., Chang, L.-H., Shibata, K., Sasaki, Y., \& Watanabe, T. (2012). Resetting capacity limitations revealed by long-lasting elimination of attentional blink through training. Proceedings of the National Academy of Sciences, 109(30), 12242-12247.

Colzato, L. S., Spapé, M. M., Pannebakker, M. M., \& Hommel, B. (2007). Working memory and the attentional blink: Blink size is predicted by individual differences in operation span. Psychonomic bulletin \& review, 14(6), 1051-1057.

Cornblatt, B. A., Risch, N. J., Faris, G., Friedman, D., \& Erlenmeyer-Kimling, L. (1988). The Continuous Performance Test, identical pairs version (CPT-IP): I. New findings about sustained attention in normal families. Psychiatry research, 26(2), 223-238. 
352 Correa, Á., Lupiáñez, J., Madrid, E., \& Tudela, P. (2006). Temporal attention enhances early 353 visual processing: A review and new evidence from event-related potentials. Brain 354 research, 1076(1), 116-128.

355 Dinges, D. F., \& Powell, J. W. (1985). Microcomputer analyses of performance on a portable, 356 simple visual RT task during sustained operations. Behavior research methods, instruments, \& computers, 17(6), 652-655.

Dux, P., \& Marois, R. (2009). The attentional blink: A review of data and theory. Attention, Perception, \& Psychophysics, 71(8), 1683-1700. doi: 10.3758/APP.71.8.1683

Hommel, B., \& Akyürek, E. G. (2005). Lag-1 sparing in the attentional blink: Benefits and costs of integrating two events into a single episode. The Quarterly Journal of Experimental Psychology Section A, 58(8), 1415-1433.

Intriligator, J., \& Cavanagh, P. (2001). The spatial resolution of visual attention. Cognitive Psychology, 43(3), 171-216. doi: 10.1006/cogp.2001.0755

The jamovi project (2019). jamovi. (Version 1.1) [Computer Software]. Retrieved from https://www.jamovi.org.

JASP team (2019). JASP (Version 0.11.1) [Computer software]. Retrieved from https://jasp$\underline{\text { stats.org }}$

Kelly, A. J., \& Dux, P. E. (2011). Different attentional blink tasks reflect distinct information processing limitations: An individual differences approach. Journal of Experimental Psychology: Human Perception and Performance, 37(6), 1867.

Kleiner, M., Brainard, D., Pelli, D., Ingling, A., Murray, R., \& Broussard, C. (2007). What's new in Psychtoolbox-3. Perception, 36(14), 1-16. 
374 Kruschke, J. K. (2013). Bayesian estimation supersedes the $t$ test. Journal of Experimental 375 Psychology. General, 142(2), 573-603. https://doi.org/10.1037/a0029146

376 Langner, R., \& Eickhoff, S. B. (2013). Sustaining attention to simple tasks: a meta-analytic

377

378

379

380

381

382

383

384

385

386

387

388

389

390

391

392

393 review of the neural mechanisms of vigilant attention. Psychological bulletin, 139(4), 870-900. doi: $10.1037 / \mathrm{a} 0030694$

Los, S. A., Knol, D. L., \& Boers, R. M. (2001). The foreperiod effect revisited: Conditioning as a basis for nonspecific preparation. Acta Psychologica, 106(1-2), 121145. https://doi.org/10.1016/S0001-6918(00)00029-9

Lunau, R., \& Olivers, C. N. L. (2010). The attentional blink and lag 1 sparing are nonspatial. Attention, Perception, \& Psychophysics, 72(2), 317-325. doi: 10.3758/app.72.2.317

Maclean, M. H., \& Arnell, K. M. (2011). Greater attentional blink magnitude is associated with higher levels of anticipatory attention as measured by alpha event-related desynchronization (ERD). Brain Res, 1387, 99-107. doi: 10.1016/j.brainres.2011.02.069

MacLean, M. H., Arnell, K. M., \& Cote, K. A. (2012). Resting EEG in alpha and beta bands predicts individual differences in attentional blink magnitude. Brain Cogn, 78(3), 218229. doi: $10.1016 /$ j.bandc.2011.12.010

Martens, A., Munneke, J., Smid, H., \& Johnson, J. (2006). Quick minds don't blink: Electrophysiological correlates of individual differences in attentional selection. Journal of cognitive neuroscience, 18(9), 1423-1438.

Martens, S., \& Johnson, A. (2005). Timing attention: Cuing target onset interval attenuates the attentional blink. Memory \& Cognition, 33(2), 234-240. doi: 10.3758/bf03195312

Martin, E. W., \& Shapiro, K. L. (2008). Does failure to mask T1 cause lag-1 sparing in the attentional blink? Perception \& Psychophysics, 70(3), 562-570. doi: 10.3758/pp.70.3.562 
397 Pelli, D. G. (1997). The VideoToolbox software for visual psychophysics: Transforming 398 numbers into movies. Spatial vision, 10(4), 437-442.

399 Potter, M. C., \& Levy, E. I. (1969). Recognition memory for a rapid sequence of pictures. $400 \quad$ Journal of experimental psychology, 81(1), 10-15. doi: 10.1037/h0027470

401 Potter, M. C., Wyble, B., Hagmann, C. E., \& McCourt, E. S. (2013). Detecting meaning in 402 RSVP at $13 \mathrm{~ms}$ per picture. Attention, Perception, \& Psychophysics, 1-10. doi: $10.3758 / \mathrm{s} 13414-013-0605-\mathrm{z}$

404 405

406

407

408

409

410

411

412 413

414 415 416 417 418

Quintana, D. S., \& Williams, D. R. (2018). Bayesian alternatives for common null-hypothesis significance tests in psychiatry: A non-technical guide using JASP. BMC Psychiatry, 18. https://doi.org/10.1186/s12888-018-1761-4

Raymond, J. E., Shapiro, K. L., \& Arnell, K. M. (1992). Temporary suppression of visual processing in an RSVP task: An attentional blink? Journal of Experimental Psychology: Human Perception and Performance, 18(3), 849.

Raz, A. (2004). Anatomy of attentional networks. The Anatomical Record Part B: The New Anatomist, 281B(1), 21-36. doi: 10.1002/ar.b.20035

Shapiro, K. L., Raymond, J. E., \& Arnell, K. M. (1997). The attentional blink. Trends in cognitive sciences, 1(8), 291-296. doi: 10.1016/S1364-6613(97)01094-2

Taatgen, N. A., Juvina, I., Schipper, M., Borst, J. P., \& Martens, S. (2009). Too much control can hurt: A threaded cognition model of the attentional blink. Cognitive psychology, 59(1), 129.

Tang, M. F., Badcock, D. R., \& Visser, T. A. (2014). Training and the attentional blink: Limits overcome or expectations raised? Psychonomic bulletin \& review, 2l(2), 406-411. 
419 Visser, T. A. W. (2015). Expectancy-based modulations of lag-1 sparing and extended sparing

420 during the attentional blink. Journal of Experimental Psychology: Human Perception and

$421 \quad$ Performance, 41(2), 462.

422 Visser, T. A. W., Tang, M. F., Badcock, D. R., \& Enns, J. T. (2014). Temporal cues and the

423 attentional blink: A further examination of the role of expectancy in sequential object

424 perception. Attention, Perception, \& Psychophysics, 76(8), 2212-2220. doi:

425 $10.3758 / \mathrm{s} 13414-014-0710-7$

426

427

428

429

430 431

432

433

434

Visser, T. A. W., Zuvic, S. M., Bischof, W. F., \& Di Lollo, V. (1999). The attentional blink with targets in different spatial locations. Psychological Bulletin \& Review, 6, 432-436.

Zauner, A., Fellinger, R., Gross, J., Hanslmayr, S., Shapiro, K. L., Gruber, W., . . Klimesch, W. (2012). Alpha entrainment is responsible for the attentional blink phenomenon. NeuroImage, 63(2), 674-686. doi:10.1016/j.neuroimage.2012.06.075 


\section{Figure 1}

Attentional Blink procedure

Eight frames of an RSVP stream in an attentional blink task. Participants are asked to identify two target stimuli (numbers) within distractor stimuli (letters). The above figure displays T2 presented at a lag of 3 (i.e., three items after T1). In our experiment, the timing was exactly $100 \mathrm{~ms}$ for each stimulus (12 frames at $120 \mathrm{~Hz}$ ), as illustrated here.

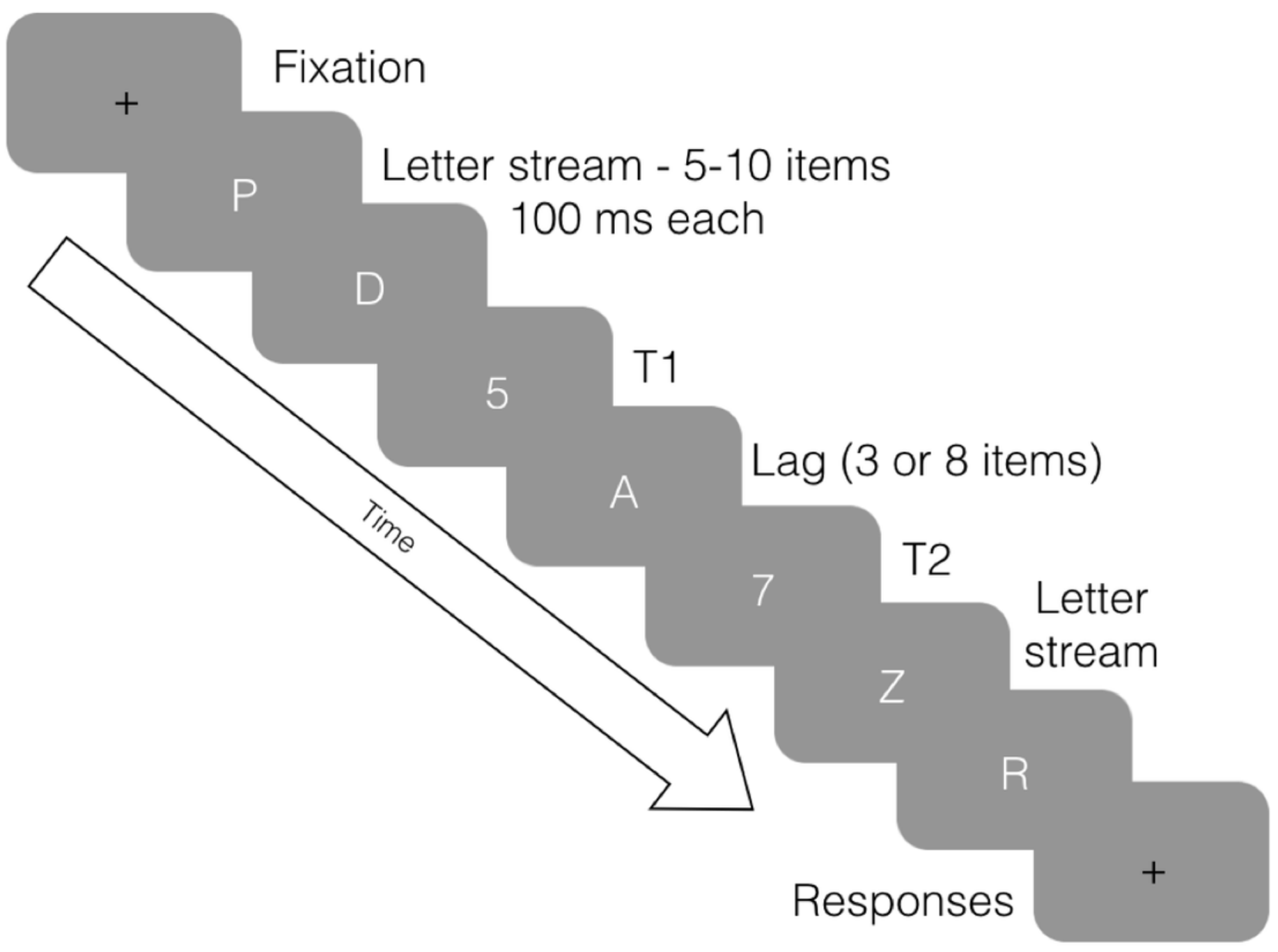


Figure 2

Typical results for an $A B$ task

This fgure illustrates typical performance on attentional blink tasks, showing lag 1 sparing with intact performance at lag 1 and impaired performance at lags 2-5. Note T2|T1 (blue line) represents correct detection of $\mathrm{T} 2$ following correct detection of $\mathrm{T} 1 ; \mathrm{T} 2$ only (red line) represents correct detection of T2 when the observer is not required to detect T1.

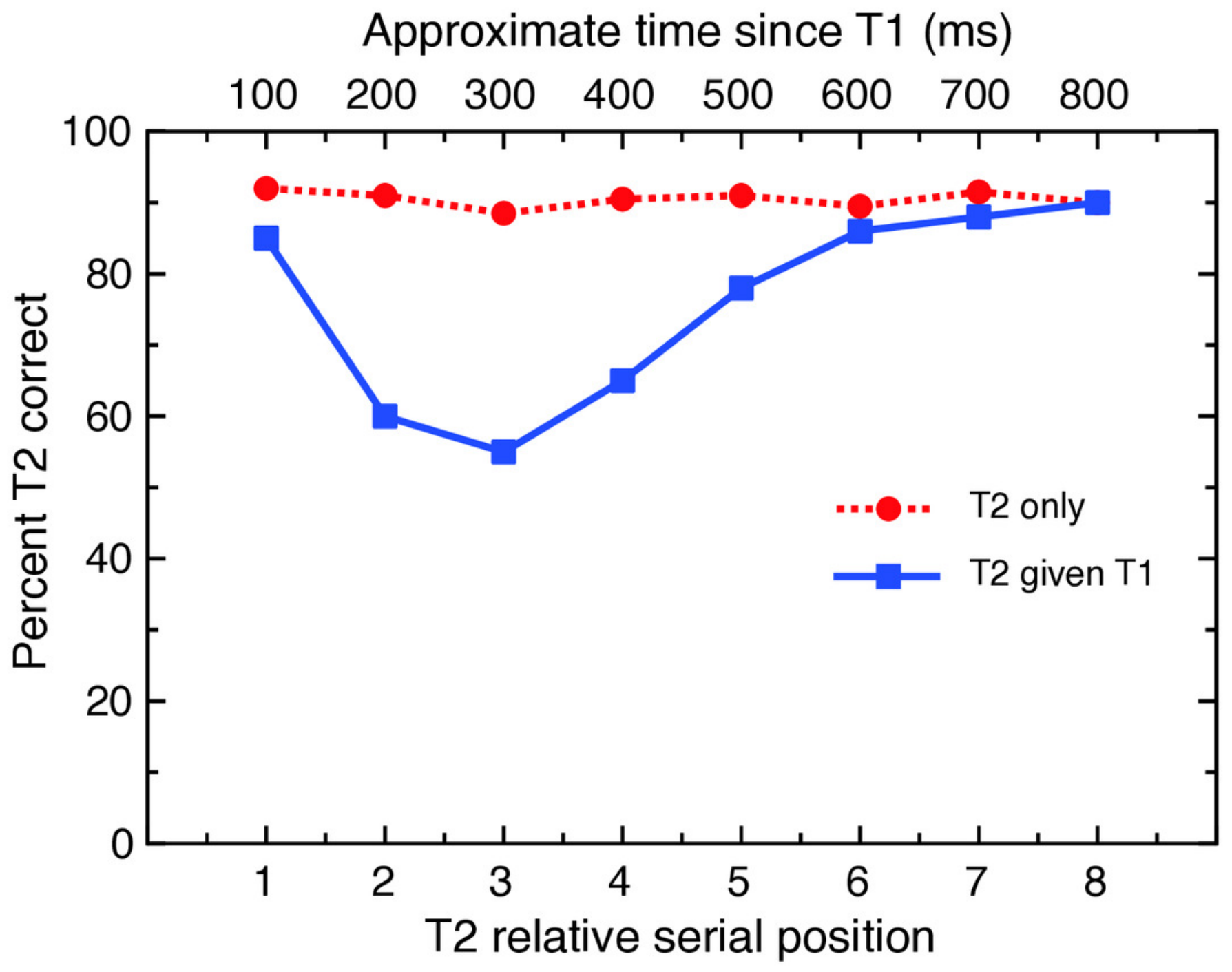


Figure 3

Accuracy and blink magnitude for random vs. fixed inter-trial intervals

T2|T1 accuracy (i.e., the proportion of T2 targets correctly identified for trials in which T1 was also correctly identified), comparing fixed and random ITI at each lag (a), and blink magnitude (b) (lag 8 - lag 3). Means are shown by black horizontal bars. Individual scores are represented by black circles, slightly jittered for clarity; colored areas represent $95 \%$ Highest Density Intervals (HDIs), calculated using R's BEST (Bayesian Estimation Supersedes the TTest) package (Kruschke, 2013), and vertical bars represent the 10th and 90th quantiles.

a)

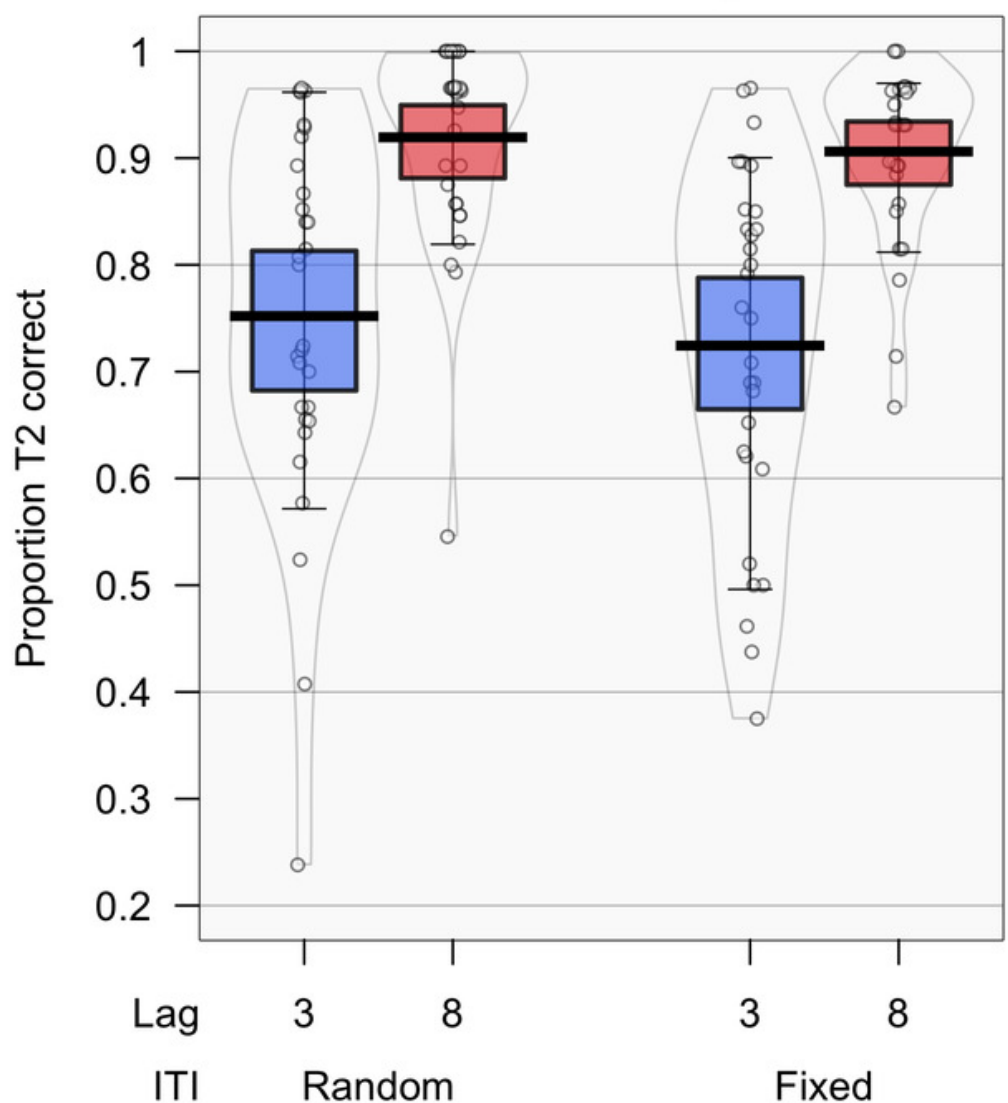

b)

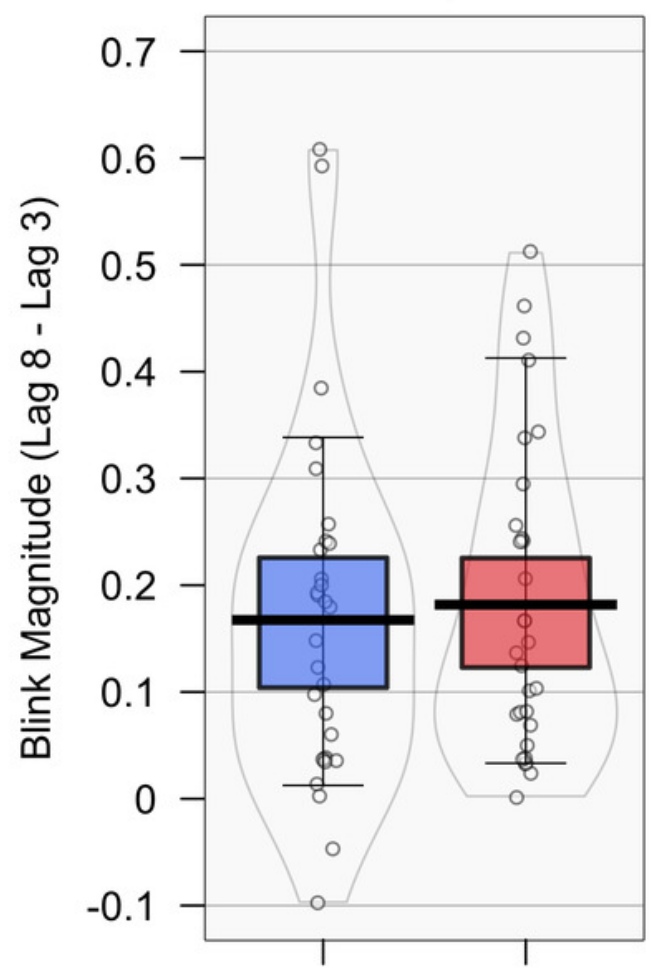

Random
Fixed 
Figure 4

Individual subjects' performance on fixed and random ITI conditions according to trial order.

Blink magnitude (lag 8 accuracy - lag 3 accuracy), comparing fixed and random ITI for the different trial order conditions (fixed ITI first and random ITI first). Individual subjects are represented by the linked points; fixed-first and random-first conditions are indicated by color.

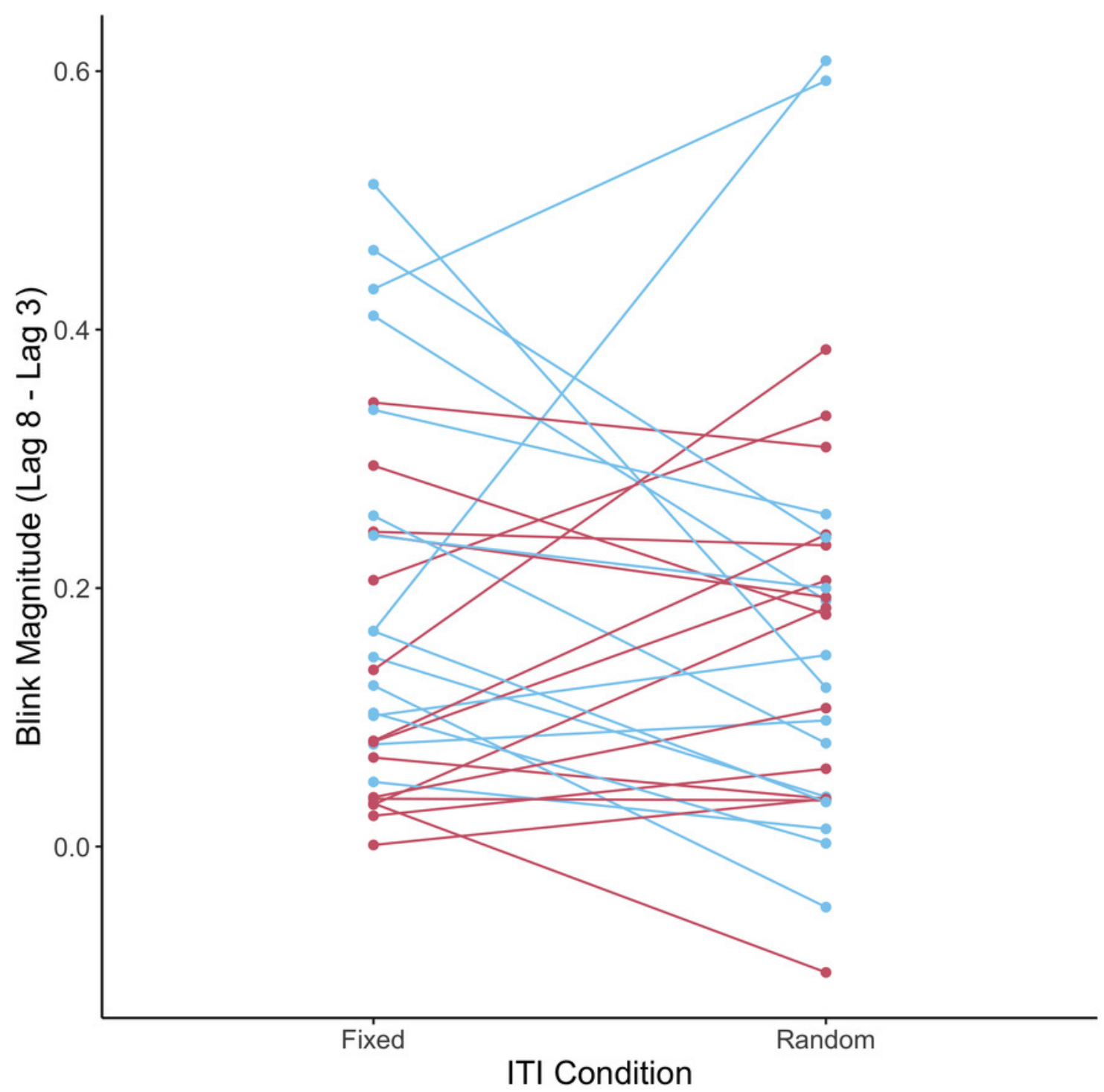

Trial Order

$\rightarrow$ Fixed, Rand

$\rightarrow$ Rand, Fixed 